Short communication:

\title{
Approach to observe folded chains of syndiotactic polystyrene on solution-grown crystals by atomic force microscopy
}

\author{
Nobuyuki Suto, Atsuhiro Fujimori, Toru Masuko *
}

Department of Polymer Science and Engineering, Faculty of Engineering, Yamagata

University, Jonan 4-3-16, Yonezawa, Yamagata, 992-8510, Japan;

Fax +81-238-26-3073; fujimori@yz.yamagata-u.ac.jp

(Received: August 12, 2003; published: October 28, 2003)

\begin{abstract}
Folded chains of syndiotactic polystyrene located on the surface of its solution-grown crystals (SGCs) have been observed on nano scale by use of atomic force microscopy (AFM). Transmission electron micrographs of the SGCs on the mesoscopic scale exhibited truncated-lozenge platelets accompanied by a sharp electron diffraction pattern of the $\beta$ "-form. AFM images of selected areas on the SGC surface indicated folded chains existing periodically on the surface with c. 1.3 to $1.4 \mathrm{~nm}$ spacing, suggesting that the polymer chains are aligned in parallel with the $\{230\}$ growth faces of SGC.
\end{abstract}

\section{Introduction}

Investigating the folding mechanism of macromolecular chains is one of the most important subjects for understanding fine structures of crystalline polymers. Chain folding is widely known to be a common feature of flexible polymers, after solutiongrown crystals (SGCs) of linear polyethylene were independently found by Keller [1], Till [2], and Fischer [3]. However, until now, few studies have succeeded in direct observations of the folded chains located on the surface of SGCs by atomic force microscopy (AFM) [4,5]. One of the difficulties comes from the fact that local mode motions (mainly internal rotations of about 10 monomer units) exist in the folded chains, which are unfavourable for improving the positional resolution in probemicroscopic observations.

Recent progress in polymer synthesis has resulted in manufacturing syndiotactic polystyrene (s-PS) [6]. This s-PS with excellent stereo-regularity has a relatively high melting temperature $\left(\approx 270^{\circ} \mathrm{C}\right)$ as compared with that of isotactic polystyrene (i-PS) [7], though both of them possess almost the same glass transition temperature of about $100^{\circ} \mathrm{C}$. SGCs of S-PS are supposed to be a suitable candidate for observing folding features since the chain has a large molecular diameter, a symmetrical shape and much higher temperatures associated with local mode relaxation.

In the present investigation, we shall try to observe directly the folding feature of s-PS on the surface of SGC by contact mode AFM measurements on nano scale. Further- 
more, the images obtained will be compared with molecular modelling results and then discussed with respect to the mechanism of chain folding.

\section{Experimental part}

s-PS pellets $\left(M_{\mathrm{w}}=375000, M_{\mathrm{w}} / M_{\mathrm{n}}=3.0\right)$ were kindly supplied by Idemitsu Petrochemical Co., Ltd., and were used without any further purification. A mixture of 2:1 (v/v) n-tetradecane/decahydronaphthalene [T/D] was utilized as the solvent for SGC preparation. This mixed solvent was first introduced by Keith et al. to prepare SGCs of i-PS [8]. A 0.005 wt.-\% solution of s-PS was prepared by dissolving a pellet in the boiling solvent $\left(234^{\circ} \mathrm{C}\right)$. The solution was then cooled down gradually to a crystallization temperature of $200^{\circ} \mathrm{C}$, and held isothermally for $2 \mathrm{~h}$. The precipitated platelets of SGC thus obtained were carefully transferred on a carbon-coated sheet mesh, followed by shadowing with $\mathrm{Au} / \mathrm{Pd}$ alloy at an angle of $27^{\circ}$. The specimen was examined by use of a Philips CM-300 electron microscope at an acceleration voltage of $200 \mathrm{kV}$.

Alternatively, a small amount of [T/D] solution involving SGC was dropped onto a newly cleaved mica surface at room temperature, and then the droplet was dried out in a desiccator at $60^{\circ} \mathrm{C}$ for several days. A scanning probe microscope (Seiko Instruments, SPA300 with SPI-3800 probe station) was employed at $23^{\circ} \mathrm{C}$ to examine the surface of as-prepared SGCs. A rectangle-shaped $\mathrm{Si}_{3} \mathrm{~N}_{4}$ tip was applied to the specimen surface in cyclic contact mode experiments with a spring contact force of $0.09 \mathrm{~N} / \mathrm{m}$. Scan rates of $0.5-1.0 \mathrm{~Hz}$ (applied force: $1.0 \mathrm{nN}$ ) were used in mesoscopic scale measurements, and rates of $16-32 \mathrm{~Hz}$ (applied force: $9.0 \mathrm{nN}$ ) on nanoscopic scale.

The $x-y$ scanner for molecular level images was calibrated by measuring a standard mica surface that exhibits a repeating unit length of $0.52 \mathrm{~nm}$ on the in-plane direction. However, the height resolution along the z-scanning direction is not good enough for evaluating nano scale sizes and to discuss the detailed surface structures on a molecular level, since the $\mathrm{Si}_{3} \mathrm{~N}_{4}$ tip only traces the outermost surface and can't invade the lower part of the chains because the spacing between folded chains is anticipated to be much smaller than the curvature of the $\mathrm{Si}_{3} \mathrm{~N}_{4}$ tip of about $20 \mathrm{~nm}$. For AFM image examination, an imaging processor was applied without any filters.

\section{Results and discussion}

Fig. 1 shows an electron micrograph and mesoscopic-scale AFM images of a SGC obtained from s-PS. In Fig. 1a, the SGC exhibits a truncated-lozenge shape. Although most of the SGCs precipitated with unavoidable heavy overgrowths and/or multilayered platelets, a few well-defined and monolayered crystals were occasionally obtained in this condition. This SGC was assigned to the $\beta$ "-form [9-11] by its electron diffraction pattern, despite the fact that s-PS is known to present a complicated polymorphism.

As illustrated in Fig. 1a, the obtuse angle between two growth faces of the SGC is estimated to be $c$. $130^{\circ}$. Comparing the transmission electron microscopy bright image with the electron diffraction (ED) pattern, we conclude that the angle of $130^{\circ}$ corresponds to that made by a diagonal cross between (230) and (230) planes. Therefore, the growth face is in parallel with the $\{230\}$ plane. These crystals have parallelogram sizes of c. $2-6 \mu \mathrm{m}$, and their lamellar thickness is estimated to be 
approximately $10 \mathrm{~nm}$ by their shadow length, the value of which can be well compared to the crystal height obtained by the cross sectional diagram of the AFM image along the dashed line in Fig. 1b.
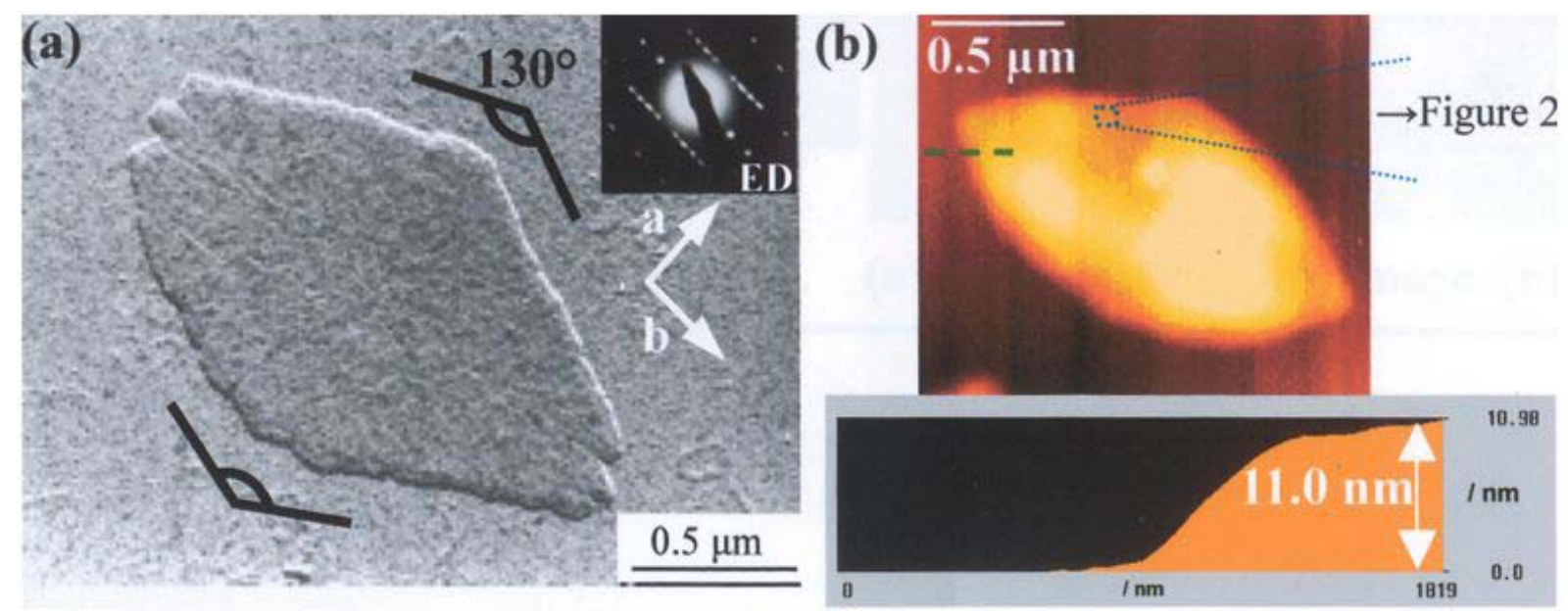

Fig. 1. Electron micrograph with ED pattern (a) and AFM image (b) with cross section diagram for a SGC of s-PS

Fig. 2 displays nano scale AFM images associated with folded chains in the truncated SGC. This just corresponds to the marked region shown in Fig. 1b, where a three-dimensional image of the surface shows an uneven waviness. Emphasis should be placed on the fact that these images have been obtained with a relatively high repulsive force of the AFM tip in order to reduce disordering or perturbing of the arrangements of folded chains. Clearly observed in this image is that the folded s-PS chains are arranged in a more or less periodical manner. The white arrow indicated in Fig. $2 b$ shows the direction perpendicular to the (230) growth face of the SGC.
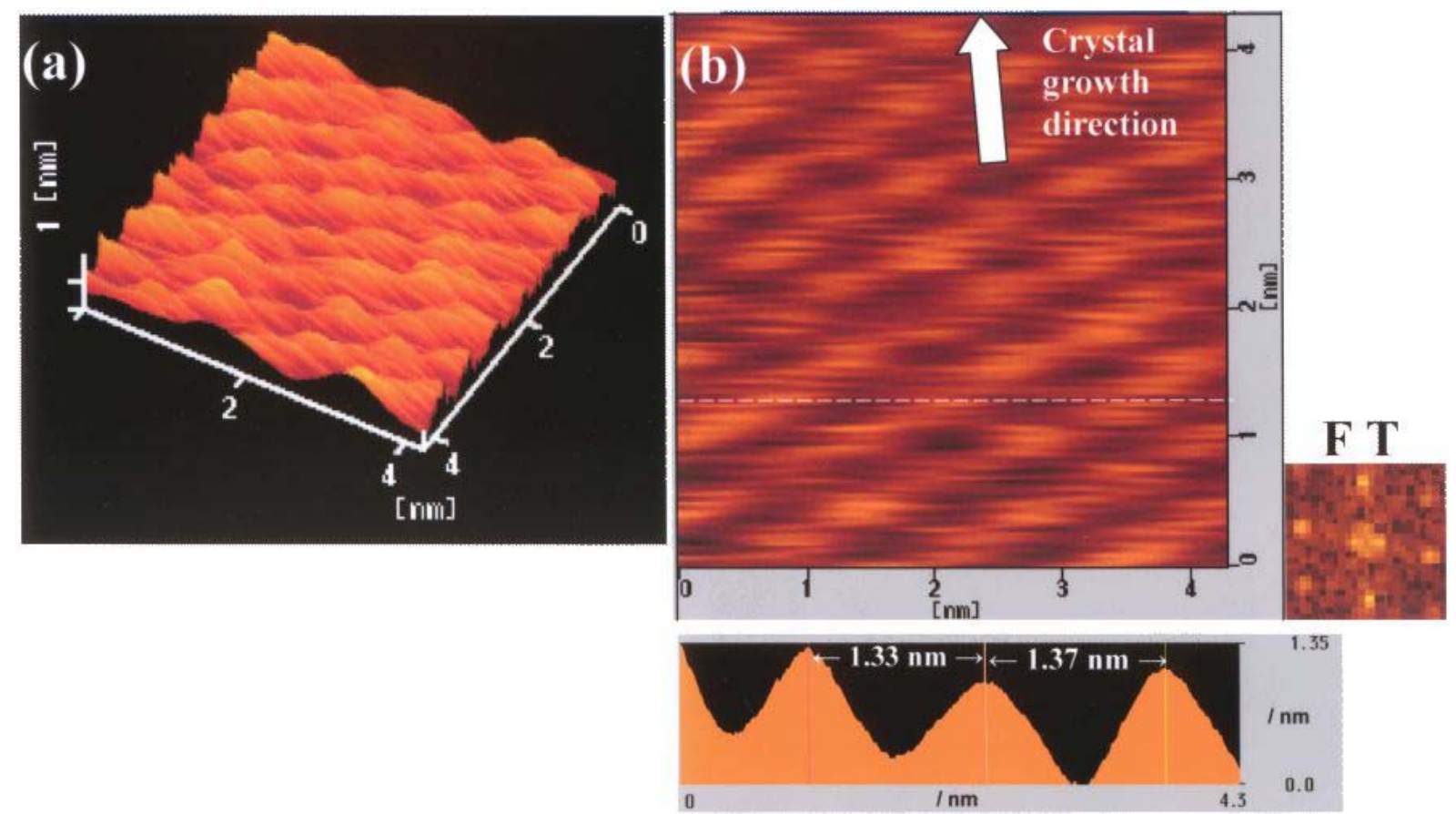

Fig. 2. AFM images of a SGC of s-PS. (a) Three-dimensional image. (b) Two-dimensional image with cross section diagram and Fourier transform image 
In addition, the image in Fig. $2 \mathrm{~b}$ yields the Fourier transform ( $\mathrm{FT}$ ) pattern as shown in the small area on the lower right-hand side, implying that surface waviness holds a kind of regularity. The FT pattern exhibits distorted-hexagonal spots related to the two-dimensionally ordered arrangement of folded chains. The cross sectional image along the dashed line in Fig. $2 \mathrm{~b}$ indicates the periodic structure aligned almost in parallel to the (230) plane, which reflects the shape of folded s-PS chains. The spacing of the individual folded units lies in the range from 1.3 to $1.4 \mathrm{~nm}$.

As described earlier, the SGC of s-PS in the present study has been assigned to the $\beta$ "-form orthorhombic system. Moreover, in Fig. $2 b$, the folded chains stand in ordered rows perpendicular to the crystal growth direction, indicating that their chain lengths are short enough for returning to the crystalline core.

In general, folded chains on polymer crystal surfaces are considered to have a variety of chain lengths; e.g., short and long chain loops coexist there. Fig. 2a tells us that the folded chains in this case are mainly composed of tight chain loops. Nano scale observations using contact mode AFM at ambient temperature are, supposedly, influenced heavily by the local mode motions due to thermal vibration. At this point, the short loops on the SGC can reduce thermal effects on the local mode because of the steric hindrance of rigid phenyl rings in the s-PS chains and their highly packed density in the crystal core. In these AFM measurements, the various images were checked by rotating the sample in constant AFM scan direction in order to avoid any artifacts. Faster or slower scanning rates brought about unclear images difficult to confirm the lattice structure on the surface.

(a)

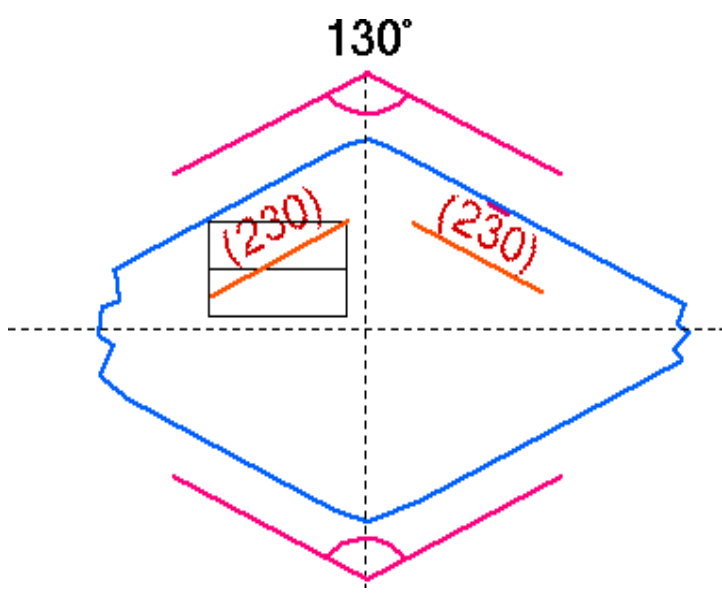

(b)

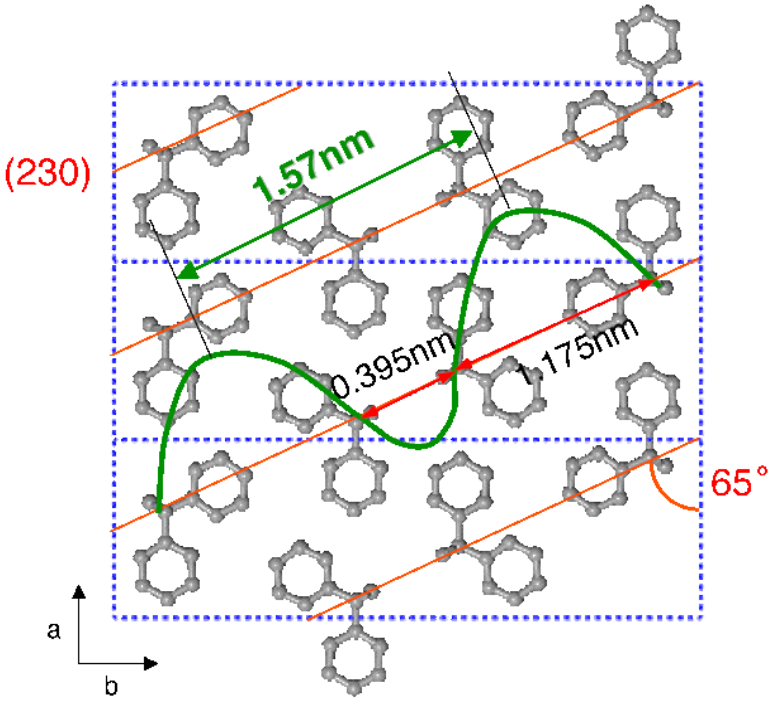

Fig. 3. Schematic representation of crystal growth faces for SGCs of s-PS (a) and the crystal structure of the $\beta "$-form of s-PS (b)

A schematic representation of the arrangement of the unit cell in the SGC is shown in Fig. 3a. The growth face parallel to (230) planes, and the angle of $130^{\circ}$ made by the crossing of a (230) and a (230) plane is consistent with the obtuse angle formed by two counter lines as observed in Fig. 1. According to the fractional coordinates of atomic positions based on the data in refs. [12,13], the crystal structure model $\left(P 2{ }_{1} 2_{1} 2_{1}\right)$ for the s-PS $\beta$ "-form was constructed with Materials Studio Ver. 2.0 
(Accelrys Inc.), as shown in Fig. 3b. The centre of $\mathrm{C}-\mathrm{C}$ bonds in the s-PS chain is aligned in the (230) planes; probably chain folding occurs along the $\{230\}$ plane. In this case, the periodical arrangements of folded chains exhibited a distance of about $1.57 \mathrm{~nm}$. This value can be expressed by the sum of two kinds of spacing between the nearest neighbours in the unit cell. Those spacings depend on the orientation of the phenyl units, $1.175 \mathrm{~nm}$ and $0.395 \mathrm{~nm}$. The value of the sum, $1.57 \mathrm{~nm}$, corresponds to the experimental finding of molecular-resolution AFM images.

In conclusion, the images of folded s-PS chains on the SGC surface have successfully been obtained by AFM observation with nano scale resolution. In those images, periodical arrangement of the folded chains was observed with about $1.3-1.4 \mathrm{~nm}$ spacing, and they were aligned in a direction parallel to the SGC growth faces.

Acknowledgement: The authors thank Professor Masatoshi Iguchi of Yamagata Univ. for helpful discussion.

[1] Keller, A.; Phil. Mag. 1957, 2, 1171.

[2] Till, P. H.; J. Polym. Sci. 1957, 24, 301.

[3] Fischer, E. W.; Z. Naturforsch. 1957, 12(a), 753.

[4] Patil, R.; Reneker, D. H.; Polymer 1994, 35, 1909.

[5] Kajiyama, T.; Ohki, I.; Takahara, A.; Macromolecules 1995, 28, 4768.

[6] Ishihara, N.; Semiya, T.; Kuramoto, M.; Uoi, M.; Macromolecules 1986, 19, 2464.

[7] Cimmino, S.; Pace, E. D.; Martuscelli, E.; Silvestre, C.; Polymer 1991, 32, 1080.

[8] Keith, H. D.; Vadimsky, R. G.; Padden Jr., F. J.; J. Polym. Sci., A-2 1970, 8, 1687.

[9] Guerra, G.; Vitagliano, V. M.; De Roza, C.; Petraccone, V.; Corradini, P.; Macromolecules 1990, 23, 1539.

[10] Tsuji, M.; Okihara, T.; Tosaka, M.; Kawaguchi, A.; Katayama, K.; MSA Bulletin 1993, 23, 57.

[11] In this case, an orthorhombic unit cell with the dimensions $a=0.90 \mathrm{~nm}$ and $b=$ $2.88 \mathrm{~nm}$.

[12] Chatani, Y.; Shimane, Y.; ljitsu, T.; Yukinari, T.; Polymer 1993, 34, 1625.

[13] De Rosa, C.; Rapacciuolo, M.; Guerra, G.; Petraccone, V.; Polymer 1992, 33, 1423. 